\title{
Time-aware Software Defined Networking for OpenFlow-based Datacenter Optical Networks
}

\author{
Hui Yang, Jie Zhang, Yongli Zhao, Yuefeng Ji \\ State Key Laboratory of Information Photonics and Optical Communications, \\ Beijing University of Posts and Telecommunications, Beijing (China) \\ Tel: 86-134-6677-4108Ｅ-mail: yanghui@bupt.edu.cn
}

Jianrui Han, Yi Lin, Shaofeng Qiu, Young Lee

Advanced Technology Division, Huawei Technologies Co., Ltd., Shenzhen (China)

Received: July 5, 2014 Accepted: November 22, $2014 \quad$ Published: December 27, 2014

DOI: 10.5296/npa.v6i4.5922

URL: http://dx.doi.org/10.5296/npa.v6i4.5922

\begin{abstract}
Data center networks are considered to make use of the computing and storage resources in data centers, which include intra-datacenter and inter-datacenter networks. Both of them will depend on the optical networking due to its advantages, such as low latency, high bandwidth, and low energy consumption. Data center interconnected by flexi-grid optical networks is a promising scenario to allocate spectral resources for applications in a dynamic, tunable and efficient control manner. Due to the high burstiness and high-bandwidth characteristics of the services, optical interconnect in intra-datacenter networks has attracted much attention compared with inter-datacenter network. Many datacenter applications in the environment require lower delay and higher availability with the end-to-end guaranteed quality of service. In this paper, we propose a novel time-aware software defined networking (TaSDN) architecture for OpenFlow-based datacenter optical networks, by introducing a time-aware service scheduling (TaSS) strategy. TaSDN can arrange and accommodate the applications with required QoS considering the time factor, and enhance the responsiveness to quickly provide for datacenter demand. The overall feasibility and efficiency of the proposed architecture are experimentally verified on our testbed with OpenFlow-based intra-datacenter and inter-datacenter optical networks.
\end{abstract}

Keywords: software defined network, OpenFlow, time-aware, data center network, optical interconnect, flexi-grid optical network 


\section{Introduction}

Due to the rapid evolving of cloud computing and high-bitrate video services, datacenter applications have attracted much attention of service providers and network operators. With the diversity and hugeness of the services, the high-performance network-based datacenter applications have presented the high burstiness and high-bandwidth characteristics, especially for the super-wavelength application beyond 100Gbps [1]. Flexi-grid optical networking, which is capable of ultrahigh capacity, distance adaption, and low power density, represent a potentially solution for overcoming emerging datacenter network bottlenecks. A novel optical orthogonal frequency division multiplexing (OFDM)-based architecture for data center networks which can provide high spectral efficiency and high energy efficiency is presented [2]. Inter-datacenter networking architecture, algorithm and control plane is also addressed in flexi-grid optical networks [3]. Advantages of software defined optical networking for highly virtualized datacenters are discussed, including lower power, improved scalability and port density [4].

Compared with inter-datacenter networks, optical interconnect in intra-datacenter networks is a more pressing need and promising scenario to accommodate these applications in a dynamic, flexible and efficient manner [5]. Additionally, various datacenter applications (e.g., service migration and backup) require lower delay and higher availability with corresponding level end-to-end guaranteed quality of service (QoS) [6]. Many studies have been focused on the datacenter interconnection architecture and equipment [7-9]. For instance in work [7], arrayed waveguide grating router (AWGR)-based interconnect architecture is proposed with a distributed all-optical control plane, which can guarantee low latency and high-throughput at high traffic load when the packet transmission time is sufficiently. The authors in [8] study a novel network on-and-off chip approach for highly efficient and transparent intra-datacenter communications to achieve the hitless adaptation between Ethernet and time shared optical network (TSON). The reference [9] proposes efficient scheme to all-optically inter-networking the pods of a datacenter, which offloads heavy inter-pod traffic onto an optical multi-ring burst network. However, few of researches pay attention to the time feature of application to guarantee the services delivery with various QoS in intra-datacenter networks from the service's point of view. Recently, as a centralized software control architecture, the software defined networking (SDN) enabled by OpenFlow protocol has gained popularity by supporting programmability of network functionalities and protocols [10-12], which can provide maximum flexibility for the operators and make a unified control over various resources for the joint optimization of services with a global view [13-15]. Therefore, it is necessary to apply SDN/OpenFlow technique to globally control network and application resources in optical intra-datacenter interconnect.

The SDN architecture with cross stratum optimization (CSO) between optical network and application stratum resources in inter-datacenter networks is proposed to partially meet the QoS requirement in our previous work [16-19]. On the basis of it, this paper proposes a novel time-aware software defined networking (TaSDN) architecture in OpenFlow-based datacenter optical networks for service migration, by introducing a time-aware service scheduling (TaSS) strategy. TaSDN can arrange and accommodate the applications with 
required QoS considering the time factor, and enhance the responsiveness to quickly provide for datacenter demand. The overall feasibility and efficiency of the proposed architecture are experimentally verified on our testbed with OpenFlow-based intra-datacenter and inter-datacenter optical networks in terms of blocking probability and resource occupation rate. Data center networks, including intra-datacenter and inter-datacenter networks, are focused on in this paper. SDN is deployed in both two networks because of its unified and flexible control advantages.

The rest of this paper is organized as follows. In Section II, we propose the novel TaSDN based datacenter network architecture and builds functional models. Then intra-datacenter optical interconnection architecture and inter-datacenter optical network architecture are described respectively. The TaSS strategy under this architecture is introduced in Section III. Finally, we describe the testbed and present the experimental results and analysis in section IV and conclude the paper in section V.

\section{TaSDN based Datacenter Network Architecture}

In order to improve the control efficiency of data center networks, TaSDN based control architecture is designed as shown in Fig. 1. Different OpenFlow controllers have been developed for different resources, which is composed of intra-datacenter processing recourse and inter-datacenter networking resources. The latter includes flexi-grid optical network resource in this study. All these kinds of resources are software-defined with OpenFlow and serve for datacenter application. Then network controller and application controller implemented by OpenFlow controller can work together. Application plane can provide users with various services through user and application interface (UAI). Also it is served through application and controller interface (ANI). The architecture of intra-datacenter and inter-datacenter networks is discussed in detail as follows.

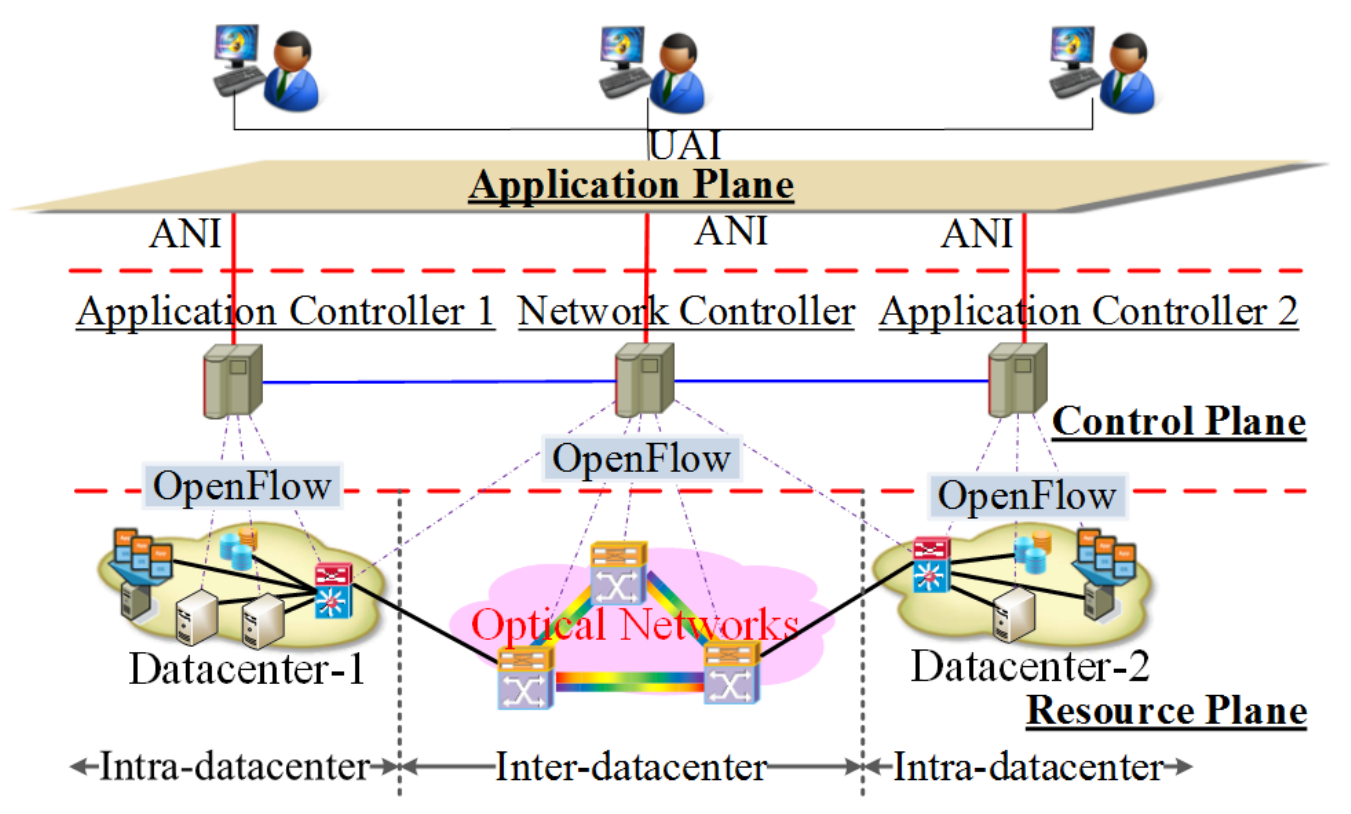

Figure 1. TaSDN based datacenter network architecture. 


\subsection{Intra-datacenter Optical Interconnection Architecture}

The TaSDN architecture for OpenFlow-based intra-datacenter optical interconnect is illustrated in Fig. 2(a). Three levels of optical switches, including top-of-rack (ToR), aggregation and core optical switches, are used to interconnect datacenter servers, which deployed application stratum resources (e.g., CPU and storage). Each stratum resources are software defined with OpenFlow and controlled by application controller (AC) and network controller (NC) respectively in a unified manner. To control intra-datacenter networks for service migration with extended OpenFlow protocol (OFP), OpenFlow-enabled optical switches with the OFP agent software are required, which are referred to as OF-OS. The motivations for the TaSDN architecture in intra-datacenter networks are twofold. Firstly, TaSDN can emphasize the cooperation between AC and NC to schedule datacenter services based on different time sensitivity requirements reasonably and optimize application and network stratum resources utilization with TaSS strategy. Secondly, based on the burstiness of intra-datacenter application, TaSDN can implement quickly burst service provisioning through centralized TaSDN control and essential procedure.

The OpenFlow-enabled switching fabric of intra-datacenter is shown in Fig. 2(b). The basic responsibilities and interactions among these functional modules are described as follows. The responsibility for the AC is concerned with monitoring and maintaining application stratum resources in datacenter servers for TaSDN, while NC sustains optical network stratum information abstracted from physical network and lightpath provisioning in intra-datacenter optical networks. Once datacenter service request arrives, AC arranges it with TaSS strategy based on time sensitivity and realizes CSO of application and network resource stored in internal database and sent the result to NC. In NC, abstraction network and physical network control are involved in. The former manages the abstracted network and provides abstracted information to $\mathrm{AC}$, while the latter is responsible for monitoring and controlling the tunable optical module. On receiving request from $\mathrm{AC}$, the end-to-end lightpath can be calculated and provisioned by using extended OFP in NC. Note that, burst data flow can be sent to high level optical switch by fast tunable laser (FTL) and received at burst mode receiver (BMR) in ToR switch. The aggregation and core switching fabrics utilize space and wavelength circuit-switching technologies and implemented with fast optical switch (FOS) and cyclic arrayed waveguide grating (CAWG) respectively. The OFP agent software embedded in optical module maintains optical flow table and models node information as software and maps the content to configure and control the physical hardware.

To the control of intra-datacenter networks, flow entry of OFP is extended and illustrated in Fig. 2(c). In this architecture, the rule is extended as the in/out port, intra-datacenter label (e.g., channel space, lambda and time slot) and port constraints, which are the main characteristics of intra-datacenter. The action for TaSDN of optical node mainly includes five types: add, switch, drop and configure to set up a path, and release a path. Various combinations of rule and action realize the control of optical node. The stats function is responsible for monitoring the flow property to provide service provisioning for TaSDN. 


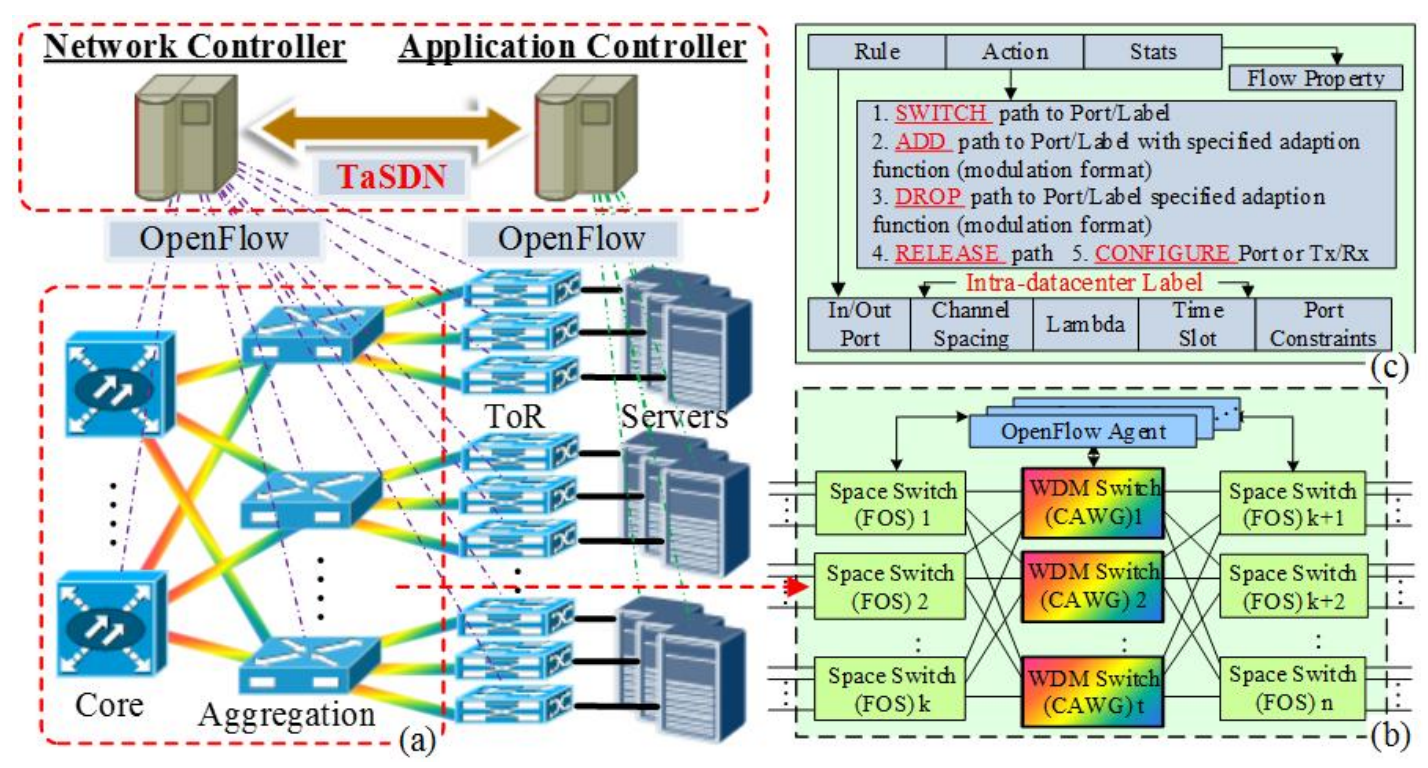

Figure 2. (a) The architecture of TaSDN in OpenFlow-based intra-datacenter networks. (b) The OpenFlow-enabled switching fabric of intra-datacenter. (c) The extension of protocol for TaSDN in intra-datacenter.

\subsection{Inter-datacenter Optical Network Architecture}

As mentioned above, flexi-grid optical networks are considered as the implementation solution of inter-datacenter networks because such networks can meet the burstiness requirement of services. The inter-datacenter optical networking architecture by flexi-grid optical networks is designed for the application scenario.

The TaSDN architecture for OpenFlow-based inter-datacenter optical network is illustrated in Fig. 3(a). The flexi-grid optical networks are used to interconnect the distributed datacenters. It follows that the network architecture mainly consists of two stratums: the optical resources stratum and application resources stratum. Each resource stratum is software defined with OpenFlow and controlled locally by network controller and application controller in a unified manner. To control the flexi-grid optical networks for inter-datacenter network with extended OFP, OpenFlow-enabled elastic optical device nodes with OFP agent software are required, which are referred to as software defined OTN (SD-OTN). The motivations for the TaSDN architecture over inter-datacenter optical network are twofold. Firstly, the TaSDN emphasizes the cooperation between AC and NC to realize software defined path (SDP) with application and spectrum elasticity through the global interworking of cross stratum resources, which can adjust the physical layer parameter (e.g., bandwidth and modulation format). Secondly, based on the different time sensitivity requirements of services reasonably, the TaSDN can schedule data center services with time elasticity to optimize the application and network resources utilization further. Based on functional architecture described above, TaSS scheme is proposed in the AC to realize the application and network stratums resources optimization through arranging the start time, transport time and corresponding transport bandwidth for services.

The functional building blocks of $\mathrm{AC}$ and $\mathrm{NC}$ and the coupling relationship between 
different modules are shown below. In NC, physical network control and abstraction network control are included. The former is responsible for discovering and controlling the spectrum bandwidth and modulation format, while the latter manages the abstracted network and provides abstracted resource information to AC. Once a datacenter service request arrives, AC decides to implement TaSS strategy of application and network resource information stored in internal database and sent the result to $\mathrm{NC}$ via application-transport interface (ATI). On receiving service request from AC, the end-to-end SDP can be calculated and provisioned by using extended OFP in NC. Note that, the modulation format of service (e.g., QPSK and 16QAM) is determined based on the length of SDP. If the SDP has short distance, more precious spectrum bandwidth can be economized due to the use of high-level modulation format. In SD-OTN, OpenFlow-enabled agent software is embedded to keep the communication between NC and optical node. The SD-OTN maintains optical flow table and modeled node information as software and maps the content to configure and control the physical hardware, which contains flexible ROADM and ODU boards shown in Fig. 3(b)-(c) respectively. For the control of flexi-grid optical networks in inter-datacenter, flow entry of OFP is extended and illustrated in Fig. 3(d). In this architecture, the rule is extended as the in/out port, flexi-grid label (e.g., central frequency and spectrum bandwidth) and ODU label (e.g., tributary slots) which are the main characteristics of flexi-grid optical networks. The action of optical node mainly includes three types: add, switch and drop. Various combinations of rule and action are used to realize the control of flexi-grid node. The stats function is responsible for monitoring the flow property to provide SDP provisioning.

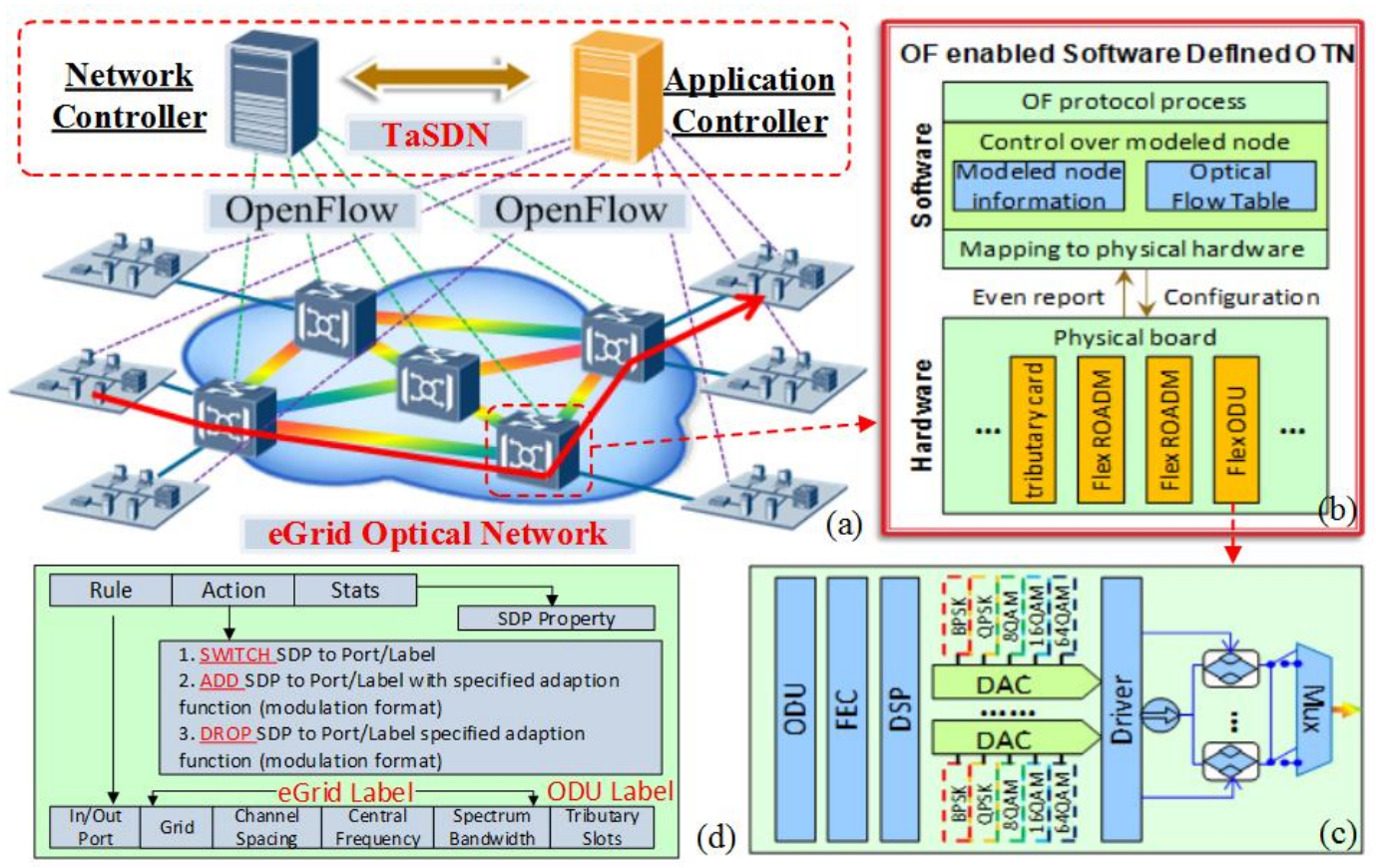

Figure 3. (a) The architecture of TaSDN in OpenFlow-based inter-datacenter networks. (b) OF-enabled SD-OTN functional models. (c) Flex ODU. (d) The extension of protocol for TaSDN in inter-datacenter. 


\section{Ml Macrothink}

\section{Service Scheduling Strategy}

In the service accommodation of datacenter optical networks, the traditional strategy can allocate the optimal application and network resources from datacenter server and corresponding lightpath at the arriving time. That may meet the following conditions, which are shown in Fig. 4 and Fig. 5. We assume there are two datacenter servers for the candidate destination node. The storage utilization of server \#1 is $80 \%$, while usage in server \#2 is $85 \%$ at the service arriving time. After a relatively short time, the services provided from two servers have changed, i.e., some services are released due to time deadline and new ones arrive. It causes that the storage utilization of server \#1 is up to $95 \%$, while usage in server \#2 reduces to $25 \%$. If according to the traditional allocation principle, it chooses the server \#1 (80\% at arriving time) as the destination node. However, if assign the resource after a period of time (within delay tolerance of user), the optimization may be better and realize the resource efficient utilization. The other instance is about the service bandwidth. Most of datacenter services use the specified bandwidth and transmit the fixed service time to achieve the overall data volume of service (i.e., the specified product of service bandwidth and time). If according to the traditional fixed bandwidth, the network cannot provide the service in case of less available bandwidth. The feasible allocation scheme can compress the bandwidth to adapt the available bandwidth and increase the service time (overall data volume of service is constant). Similarly, enhancing the provide bandwidth can complete the service as soon as possible with relatively abundant resources. The two issues are both are involved in time factor of datacenter service. Therefore, based on this feature, we propose the time-aware service scheduling strategy.
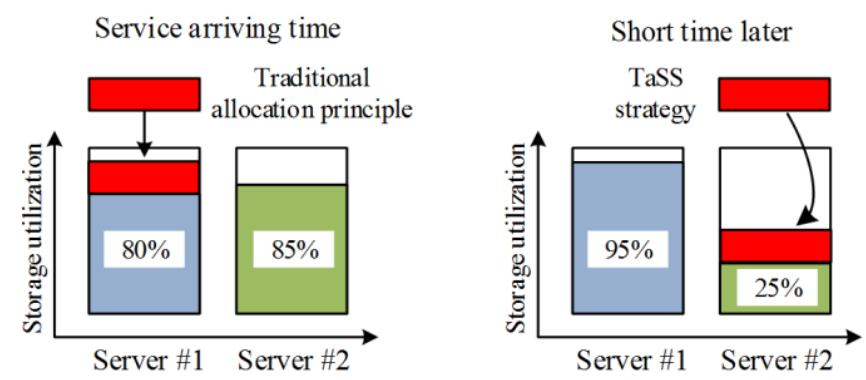

Fig. 4. Illustration of time-aware datacenter application resource allocation.

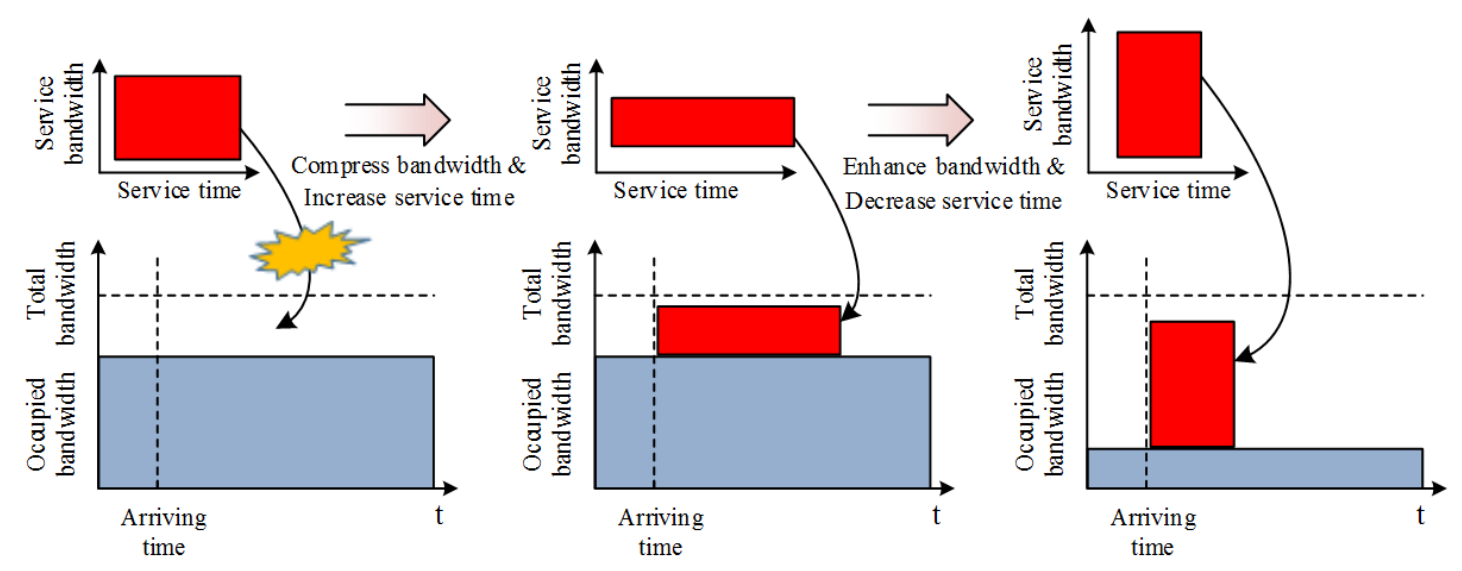

Fig. 5. Illustration of time-aware network resource allocation. 
Table 1. Notations and definitions.

\begin{tabular}{|l|l|}
\hline \multicolumn{1}{|c|}{ Symbol } & \multicolumn{1}{c|}{ Meaning } \\
\cline { 2 - 2 }$\cdot S$ & The overall volume of storage space in the destination datacenter \\
\hline$\cdot S_{r}$ & The rest volume of storage space in the destination datacenter \\
\hline$\cdot D$ & The overall data volume of service \\
\hline$\cdot B_{r}$ & The product of rest bandwidth in the lightpath \\
\hline$\cdot T$ & The tolerant latency of latency-tolerant service \\
\hline$\cdot t$ & The life time of the lightpath \\
\hline$\cdot B_{a}$ & The average transmission bandwidth in the network \\
\hline$\cdot t_{G}$ & The guard time for service accommodation \\
\hline
\end{tabular}

\subsection{Network Modeling}

The OpenFlow-based datacenter interconnect with optical networks is represented as $\mathrm{G}$ $(\boldsymbol{V}, \boldsymbol{L}, \boldsymbol{F}, \boldsymbol{A})$, where $\boldsymbol{V}=\left\{v_{1}, v_{2}, \ldots, v_{n}\right\}$ denote the set of OpenFlow-enabled optical switching nodes, $\boldsymbol{L}=\left\{l_{1}, l_{2}, \ldots, l_{n}\right\}$ indicate the set of bi-directional fiber links between nodes in $\boldsymbol{V} . \boldsymbol{F}=$ $\left\{\omega_{1}, \omega_{2}, \ldots, \omega_{F}\right\}$ is the set of wavelengths on each fiber link and $\boldsymbol{A}$ denotes the set of datacenter servers, while $N, L, F$ and $A$ represent the number of network nodes, the links, the wavelengths and datacenter nodes respectively. For each service request from source node $s$, it requires overall data volume of service $D$ and storage space $S$. We divide the services into burst latency-sensitive and latency-tolerant service. The former needs service accommodation immediately and the $i$ th request is described as $S R_{i}(s, D, S)$, while the latter contains the arriving time $t_{c}$ and tolerant latency $T$, and $i$ th service request can be denoted as $S R_{i}\left(s, D, S, t_{c}, T\right)$. The service request $S R_{i+1}$ will arrive after connection demand $S R_{i}$ in time order. In addition, some requisite notations and their definitions are listed as Table 1.

\subsection{Time-aware Service Schedule Strategy}

Based on the functional architecture described, we present a novel time-aware service scheduling (TaSS) strategy to be implemented in application controller to realize datacenter service schedule with time sensitivity requirement. For the incoming service demand, we analyze the delay sensitivity of each service and then divide them into burst latency-sensitive and latency-tolerant service, which contains flow volume and tolerant latency. For the latency-tolerant service, existing datacenter servers and corresponding lightpaths can be searched whether they have enough volume of storage space $S_{r}$ and the product of rest bandwidth $B_{r}$ and life time $t$ compared with the arriving service. If the $S_{r} \geq S$ and $B_{r} * t \geq D$, it chooses the one with minimum CSO factor [16] to provision. If there is no enough resources to accommodate, the service waits for it until exceed $\max$ tolerant latency, i.e., $t=T-D / B_{a}-t_{G}$, which considers the average transmission bandwidth $B_{a}$ and guard time $t_{G}$. Then new lightpath will be computed with cross stratum optimization to accommodate the service. For the latency-sensitive request, we find the available resources in existing candidate with the same principle. The path with minimum transmission time from existing 
paths can be prepared to provision. Once there is no available ready-made path, the TaSS strategy setups new lightpath for the service immediately. To realize the quick response for service provisioning, the release lightpath procedure is reckoned by service time immediately. The flowchart of TaSS strategy is shown in Fig. 6.

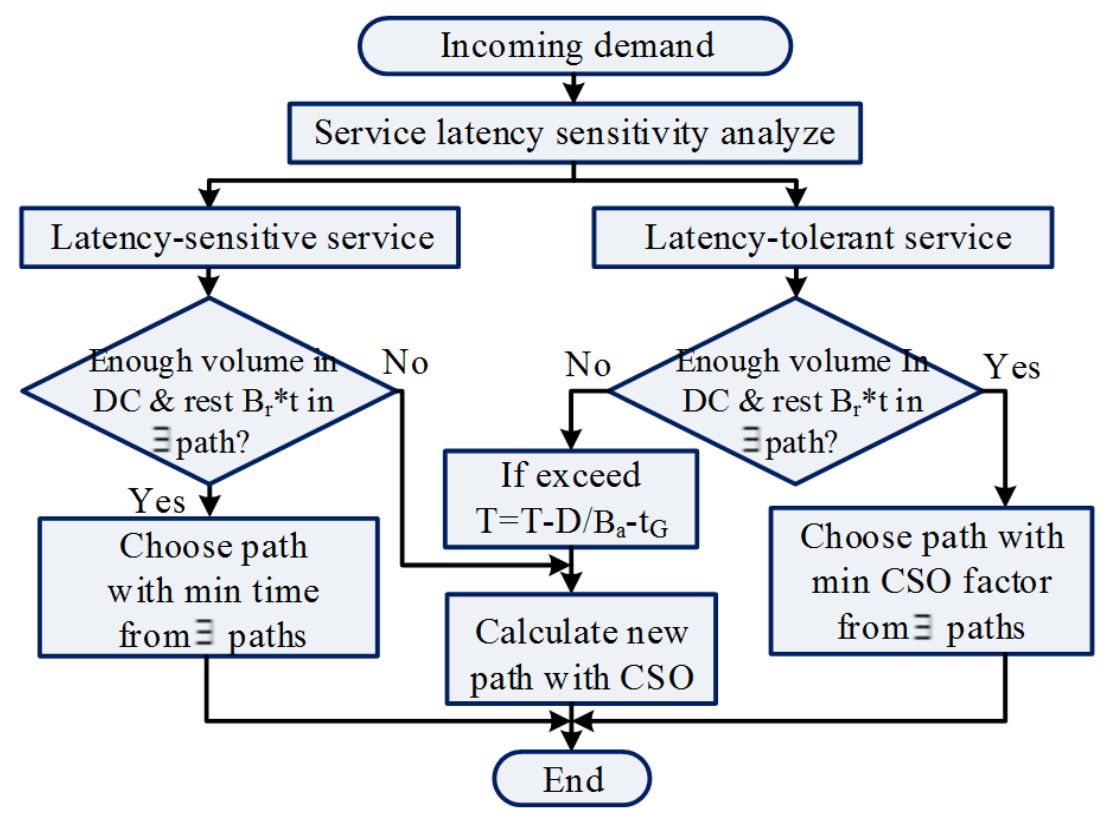

Figure 6. Flowchart of TaSS strategy.

\section{Experimental Demonstration and Results Discussion}

A testbed of datacenter networks is built, which consists of intra-datacenter and inter-datacenter. SDN is deployed on the two parts together. The testbed demonstrations are described as follows.

To experimentally evaluate of the proposed architecture, we set up TaSDN in optical intra-datacenter networks for service migration comprising both control and data planes based on our testbed, as shown in Fig. 7(a). In data plane, four optical switches are equipped with FOS, which has $25 \mathrm{~ns}$ switching time with $<4.5 \mathrm{~dB}$ insert loss. Two CAWG cards with $12.5 \mathrm{GHz}$ frequency deviation and $10.5 \mathrm{~dB}$ insert loss based on uniform loss cyclic frequency technology are deployed as in the core side. BMR with receiving power sensitivity of $-25 \mathrm{dBm}$ is built in burst mode transceiver (BMT) card with FTL, which has 98ns switching time and $2.5 \mathrm{GHz}$ frequency deviation from ITU-T standard. We develop the software OFP agent according to the API function to control the hardware through OFP. Datacenters are realized on an array of virtual machines created by VMware software running on IBM X3650 servers. Since each virtual machine has the operation system and its own CPU and storage resource, it can be considered as a real node. For OpenFlow-based TaSDN control plane, NC is assigned to support the architecture and deployed in three servers for optical module control, PCE computation and resource abstraction, while database server is used for maintaining transport resources and traffic engineering database. AC server is responsible for 


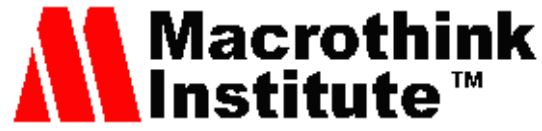

TaSS strategy with CSO agent and monitoring the application resources from datacenter servers. User plane is deployed in a server and applies the required application.

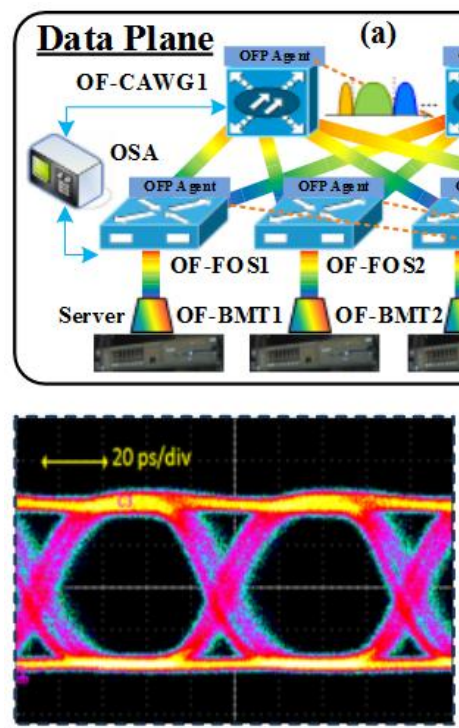

(b)

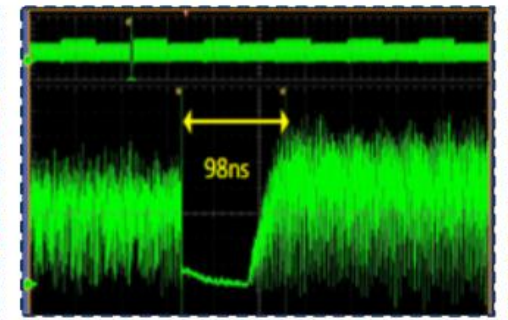

(c)
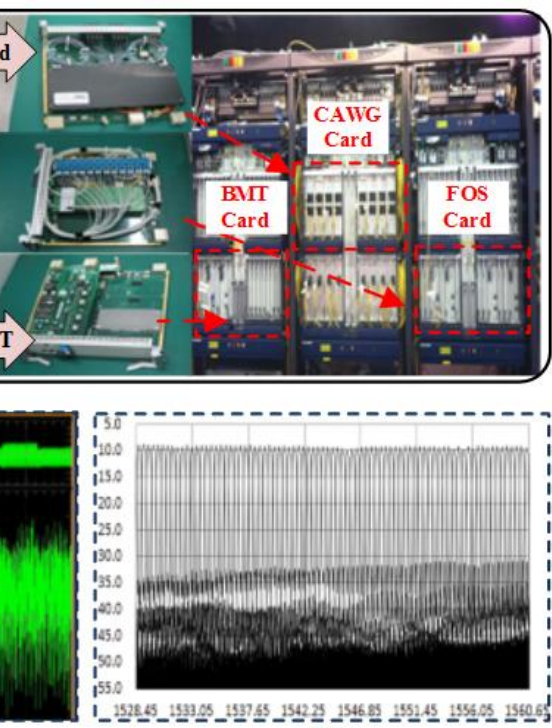

(d)

Figure 7. (a) Experimental testbed and demonstrator setup. (b, c) Eye diagram and tuning waveform of FTL. (d) Spectrum of CAWG port.

We have designed and verified experimentally lightpath provisioning in TaSDN architecture for datacenter service migration. The path and schedule for service migration is determined by AC with TaSS strategy based on various application utilizations among datacenters and current network resource, and setup from source to destination node chosen by CSO. The eye diagram and tuning waveform of FTL and spectrum of CAWG port are reflected on the filter profile, as shown in Fig. 7(b)-(d). The experimental results are further emphasized in Fig. 86(a)-(b). Figure 8(a) illustrates the capture of the OpenFlow message exchange sequence for TaSDN through Wireshark deployed in NC. As shown in Fig. 8, 10.108.50.74 and 10.108.65.249 represent the IP address of AC and NC respectively, while 10.108.50.21 and 10.108.51.22 denote the corresponding OF-OS node respectively. After the interworking between AC and NC via UDP message, AC perform TaSS strategy and then NC provision the lightpath to control all corresponding OF-OS through flow mod message along the computed path. After timing the service time, the path is released by flow mod message, while update the application usage with UDP to keep synchronization. Figure 8(b) shows a snapshot of the extended flow table modification message for lightpath provisioning, which verifies OPF extensions for TaSDN in intra-datacenter networks. We also evaluate the performance of TaSDN in intra-datacenter networks under heavy traffic load scenario through simulation, and compared TaSS with traditional CSO (TCSO) strategy [16]. The migration data volumes from datacenter node are randomly from 50Gbit to 400Gbit, which arrive to network following a Poisson process. Figure 9(a)-(b) show the comparison on the performances of two strategies. As shown, TaSS reduces blocking probability effectively comparing to TCSO, especially when the network is heavily loaded. Another phenomenon can be seen that TaSS outperforms TCSO in the resource occupation rate significantly. The 
reason is TaSS realizes global optimization considering the time schedule with various delay sensitivity requirements and adjusts the service bandwidth according to the distribution of network resources.

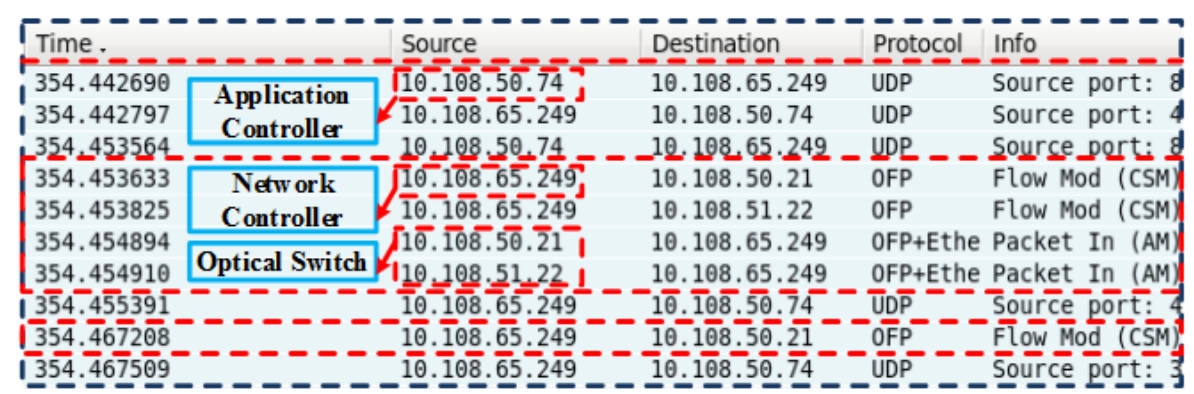

(a)

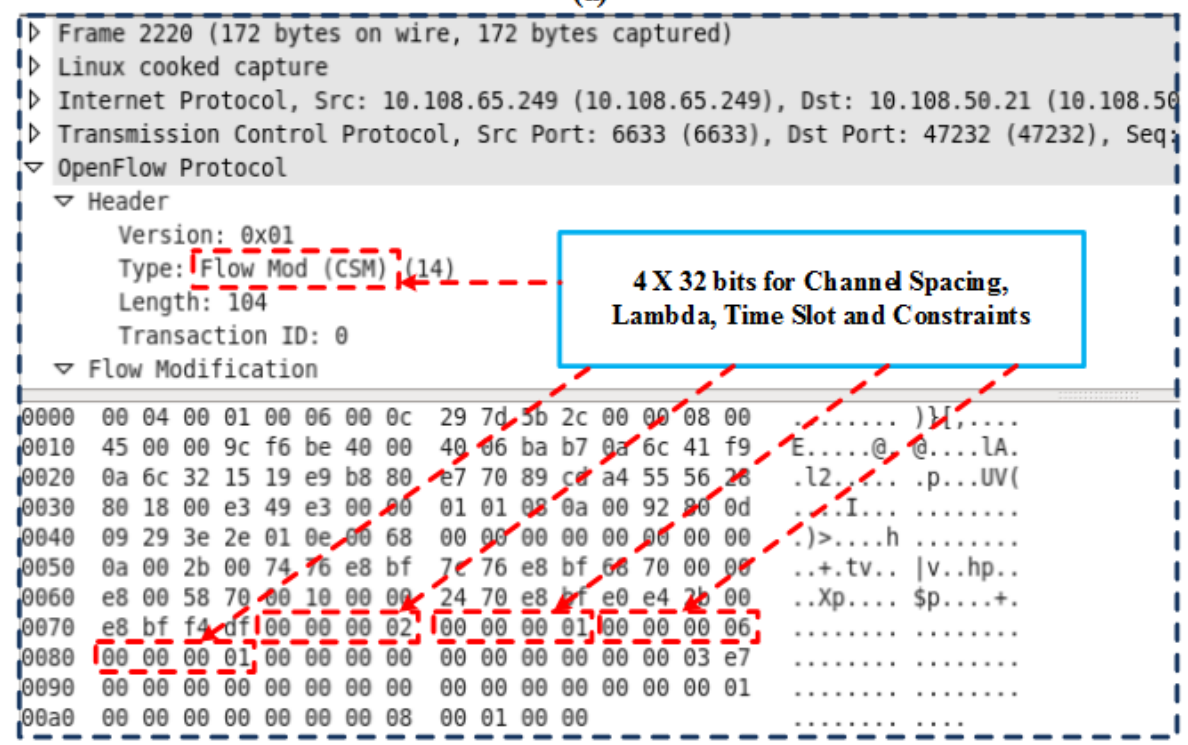

(b)

Figure 8. (a)The capture of the OpenFlow message sequence and (b) extended flow mod message for TaSDN.

To experimentally evaluate of the proposed architecture, we set up TaSDN over flexi-grid optical networks for inter-datacenter networking comprising both control and data planes based on testbed, as shown in Fig. 10(a). In data plane, OpenFlow-enabled flexible optical nodes are equipped with Huawei Optix OSN 6800, each of which comprises flex ROADM and ODU boards. Datacenters and the other nodes are also realized on virtual machines created by VMware software running on servers. Since each virtual machine has the operation system and its own independent IP address, CPU and memory resource, it can be considered as a real node. The virtual OS technology makes it easy to set up experiment topology based on the backbone of US which comprises 14 nodes and 21 links. For OpenFlow-based TaSDN control plane, the NC is assigned to support the proposed architecture and deployed in three servers for elastic spectrum control, physical layer parameter adjustment, PCE computation and resource abstraction, while the database server are responsible for maintaining traffic engineering database, management information base, connection status and the configuration of the database and transport resources. The AC server is used for CSO agent and monitoring the application resources from datacenter 


\section{Macrothink}

Network Protocols and Algorithms

ISSN 1943-3581

2014, Vol. 6, No. 4

networks with TaSS strategy. User plane is deployed in a server and applies the required application.
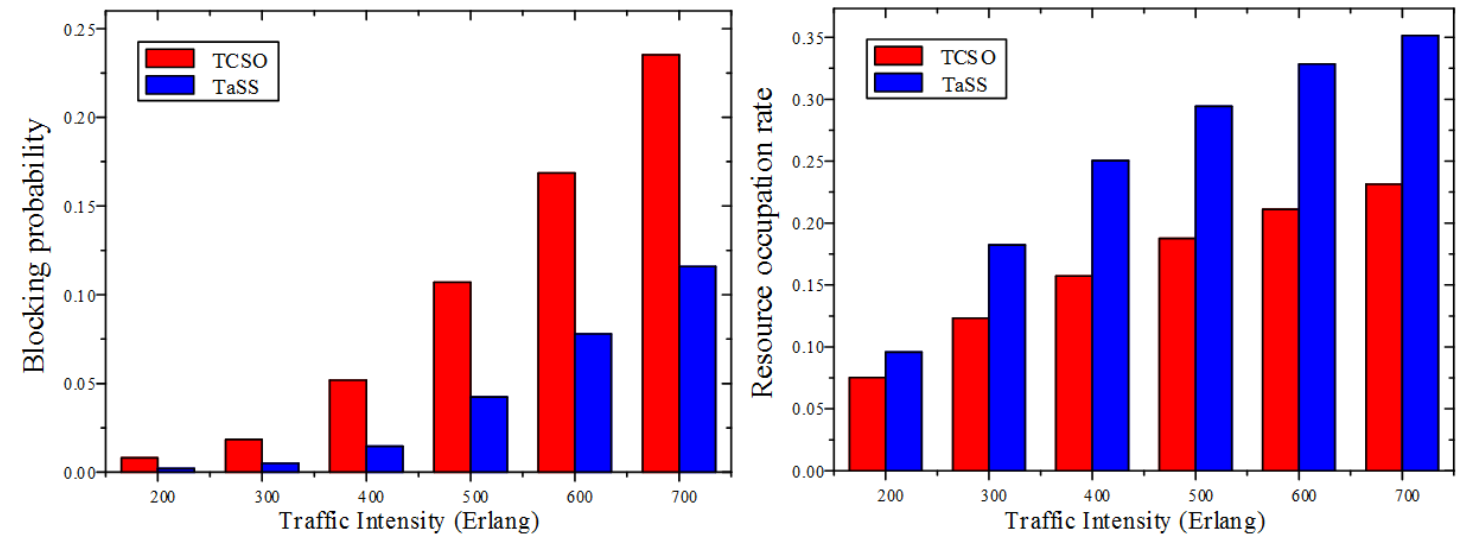

Figure 9. Comparisons on (a) blocking probability and (b) resource occupation rate between two strategies in heavy traffic load scenario.

We have designed and verified experimentally SDPs provisioning in TaSDN over flexi-grid optical networks for datacenter service migration. The experimental results are shown in Fig. 10(b)-(d). The destination datacenter is determined by AC with TaSS strategy based on various application utilizations among datacenters and current network resource, and the SDP for the service migration is setup from source to destination node. Additionally, the spectrum bandwidth and corresponding modulation format can be tunable according to different SDP distances. The spectrum of SDPs on the flexible link between two SD-OTNs is reflected on the filter profile, as shown in Fig. 10(b)-(c). The end-to-end SDP setup/release time is measured as well as timing performance of the AC and NC with SD-OTN nodes, which comprises the strategy processing time of controller, OFP propagation latency and software and hardware of device handle times, shown in Fig. 10(d).
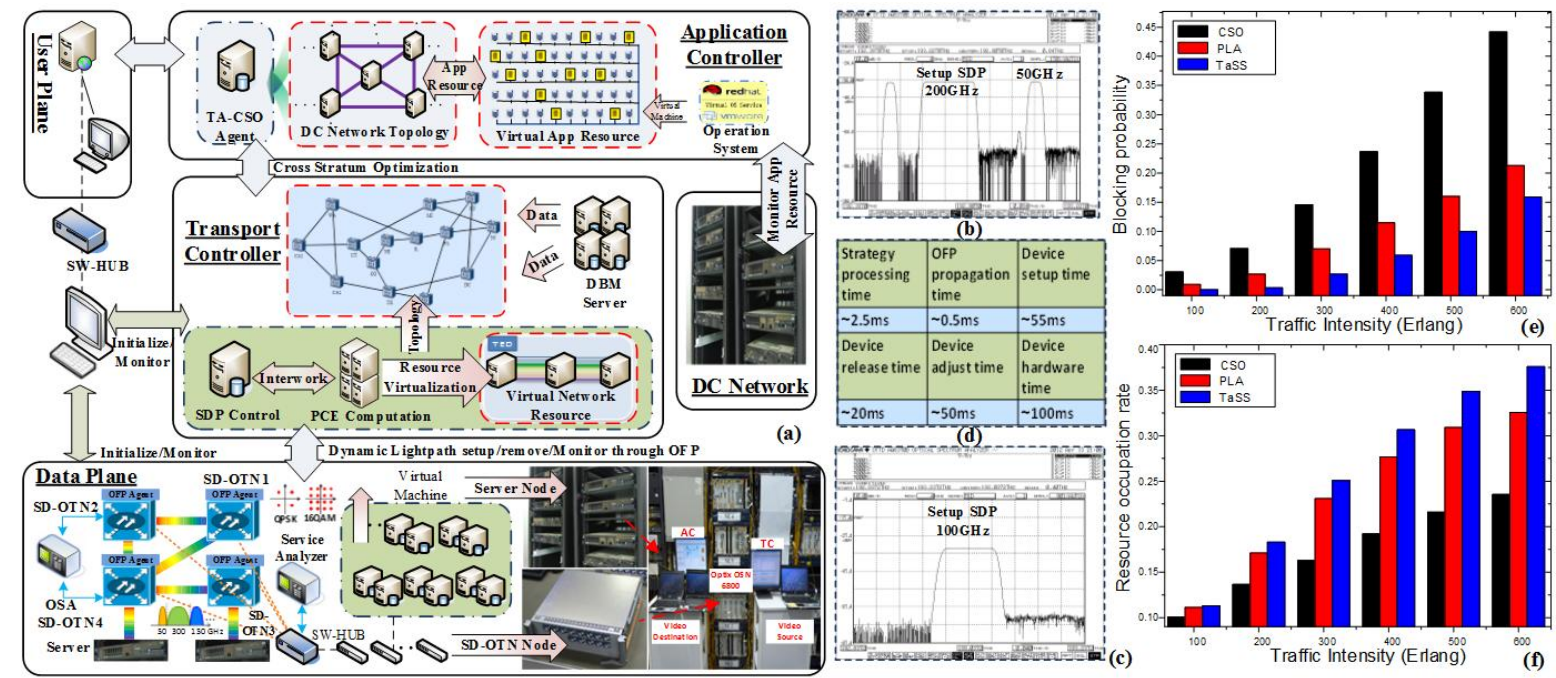

Figure 10. (a) Experimental testbed. (b, c) Bandwidth spectrum of SDPs. (d) SDP setup/release latency. (e) Blocking probability and (f) resource occupation rate under heavy traffic load scenario. 
We also evaluate the performance of TaSDN over flexi-grid networks under heavy traffic load scenario, and compared TaSS with individual CSO and physical layer adjustment (PLA) strategies. The traffic requests from OF-based flexible node to datacenter node are established with spectrum randomly from $50 \mathrm{GHz}$ to $400 \mathrm{GHz}$, where the adjustable minimal frequency slot is $12.5 \mathrm{GHz}$. They arrive to the network following a Poisson process and results have been extracted through the generation of $1 \times 10^{5}$ demands per execution. Fig. 10(e)-(f) compare the performances of three strategies in terms of blocking probability and resource occupation rate. As shown in the figure, TaSS reduces blocking probability effectively than CSO and PLA, especially when the network is heavily loaded. Another phenomenon can be seen that TaSS outperforms the other strategies in the resource occupation rate significantly. The reason is that TaSS realizes global optimization considering both application and network resources integrally, furthermore on the basis of it, economizes the spectrum resource again with choosing high-level modulation format. These results are further emphasized in Fig. 11(a)-(c). Fig. 11(a) verifies the status and destination node choice of datacenter service migration and various bandwidths of SDP in application interface of TaSDN. Fig. 11(b) illustrates the capture of the OpenFlow message exchange for TaSDN deployed in TC. Fig. 11(c) shows a snapshot of the extended flow table modification message for SDP setup, which verifies the OPF extensions for TaSDN over flexi-grid optical networks.

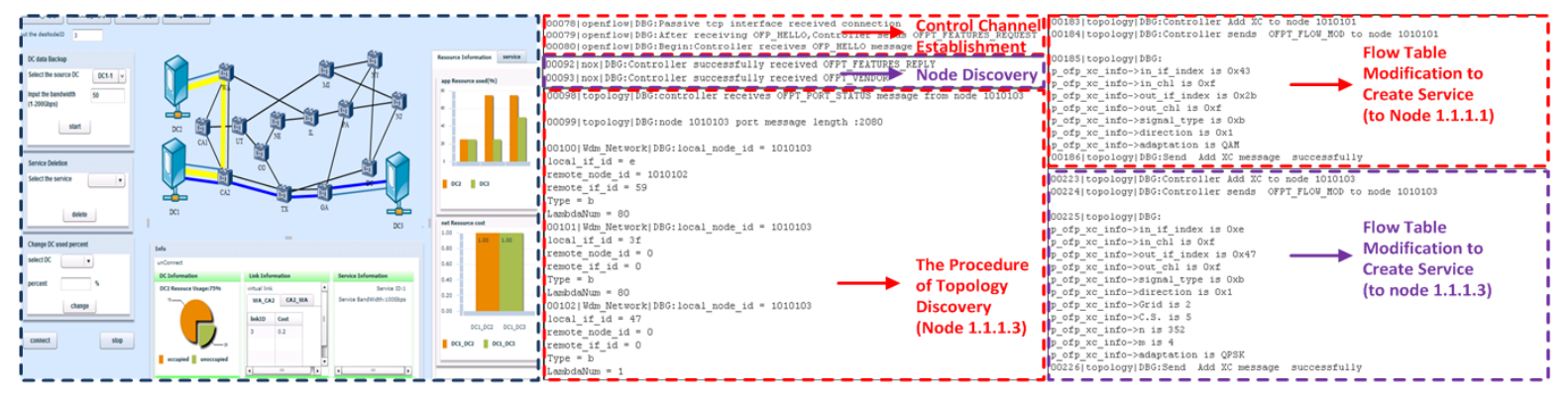

Figure 11. (a)Application graphical user interface (GUI) of testbed. (b)The capture of the OF messages for network discovery. (c) The capture of extended flow table message for SDP setup

\section{Conclusion}

Datacenter server resources will become more and more important in the future information society. How to complete the efficient allocation of datacenter resources will be the main decision factor of cost saving. Optical networking can be considered as feasible solution for both intra-datacenter and inter-datacenter networks due to the feature of high capacity and low energy consumption. The software defined networking can improve the allocation efficiency of application and network resource. This paper presents a TaSDN architecture in OpenFlow-based datacenter optical network for service migration. Additionally, the TaSS strategy is introduced for TaSDN in the proposed architecture. The main extended OpenFlow messages are described in this paper. The feasibility and efficiency of TaSDN are verified on our testbed built over intra-datacenter and inter-datacenter networks. We also quantitatively evaluate the performance of our approach under heavy traffic load 
scenario in terms of blocking probability and resource occupation rate, and compare it with TaSS strategy. The experimental results indicate that the TaSDN with TaSS strategy can schedule service with time sensitivity and utilize cross stratum resources effectively in datacenter networks.

Our future works for TaSDN include two aspects. One is to improve TaSS performance with dynamic parameters and consider the scalability issues of optical network. The other is to implement the network virtualization in datacenter optical network on our OpenFlow-based testbed.

\section{Acknowledgement}

This work has been supported in part by 863 program (2012AA011301), 973 program (2010CB328204), NSFC project (61271189, 61201154, 60932004), RFDP Project (20120005120019), the Fundamental Research Funds for the Central Universities (2013RC1201), and Fund of State Key Laboratory of Information Photonics and Optical Communications (BUPT).

\section{References}

[1] Kachris C., Tomkos I., "A Survey on Optical Interconnects for Data centers," IEEE Communications Surveys and Tutorials, vol. 14, 2012, pp. 1021-1036. http://dx.doi.org/10.1109/SURV.2011.122111.00069

[2] Kachris C., Tomkos I., "Energy-efficient bandwidth allocation in optical OFDM-based data center Networks,” OFC2012, Los Angeles, USA, Mar.2012.

[3] Yoo S. J B., Yin Y., Wen K., "Intra and inter datacenter networking: The role of optical packet switching and flexible bandwidth optical networking," ONDM 2012, Colchester, England, April 2012. http://dx.doi.org/10.1109/ONDM.2012.6210261

[4] Casimer D., "Optical networking in Smarter data centers: 2015 and beyond," OFC2012, Los Angeles, USA, Mar.2012.

[5] Yang H., Zhang J., Zhao Y., Ji Y., Han J., Lin Y., Qiu S., and Lee Y., "Experimental Demonstration of Time-aware Software Defined Networking for OpenFlow-based Optical Interconnect in Intra-Datacenter Networks," GLOBECOM 2013, WS-CCSNA, Dec. 2013, 1-6. http://dx.doi.org/10.1109/GLOCOMW.2013.6825027

[6] Yang H., Zhao Y., Zhang J., Wang S., Gu W., Ji Y., Han J., Lin Y., and Lee Y., "Multi-Stratum Resources Integration for OpenFlow based Data Center Interconnect [Invited]," Journal of Communications and Networks, vol. 5, no. 10, Oct. 2013, pp. A240-A248. http://dx.doi.org/10.1364/JOCN.5.00A240

[7] Proietti R., Nitta C. J., Yin Y., Akella V., and Yoo S. J. B., "Scalability and Performance of a Distributed AWGR-based All-Optical Token Interconnect Architecture," in Proc. of OFC/NFOEC 2013, OW3H.1, 1-3.

[8] Rofoee B.R., Zervas G., Yan Y., Amaya N., Qin Y., and Simeonidou D., "Network-on-and-off-Chip Architecture on Demand for Flexible Optical Intra-Datacenter Networks," in Proc. ECOC, 2012, Th.2.B.2. http://dx.doi.org/10.1364/ECEOC.2012.Th.2.B.2 [9] Deng N., Xue Q., Li M., Gong G., and Qiao C., “An Optical Multi-Ring Burst Network 
for a Data Center," in Proc. of OFC/NFOEC 2013, OTh1A.5, 1-3.

[10]Das S., Parulkar G., and McKeown N., "Unifying Packet and Circuit Switched Networks," in Proc. Globecom, 2009, pp. 1-9. http://dx.doi.org/10.1109/GLOCOMW.2009.5360777

[11]Liu L., Peng W., Casellas R., Tsuritani T., Morita I., Martínez R., Muñoz R., and Yoo S. J. B., "Design and performance evaluation of an OpenFlow-based control plane for software defined elastic optical networks with directdetection optical OFDM (DDO-OFDM) transmission," Opt. Express, vol. 22, no. 1, pp. 30-40, 2014. http://dx.doi.org/10.1364/OE.22.000030

[12]Jocha D., Kern A., and Yedavalli K., "Scalability Aspects of Centralized Control of MPLS Access/Aggregation Network," in Proc. Globecom, 2012, pp. 2634-2639. http://dx.doi.org/10.1109/GLOCOM.2012.6503514

[13]Liu L., Zhang D., Tsuritani T., Vilalta R., Casellas R., Hong L., Morita I., Guo H., Wu J., Martínez R., and Muñoz R., "Field Trial of an OpenFlow-Based Unified Control Plane for Multilayer Multi granularity Optical Switching Networks," J. Lightw. Technol., vol. 31, pp. 506-514, Feb. 2013.

[14]Channegowda M., Nejabati R., Rashidifard M., and Peng S., et al., "Experimental demonstration of an OpenFlow based software-defined optical network employing packet, fixed and flexible DWDM grid technologies on an international multi-domain testbed," Opt. Express, vol. 21, pp. 5487-5498, Mar. 2013. http://dx.doi.org/10.1364/OE.21.005487

[15]Casellas R., Martinez R., Munoz R., Liu L., Tsuritani T., and Morita I., “An integrated stateful PCE/OpenFlow controller for the control and management of flexi-grid optical networks," in Proc. OFC/NFOEC 2013, paper OW4G.2.

[16] Yang H., Zhao Y., Zhang J., Wang S., Gu W., Lin Y., and Lee Y., "Cross Stratum Optimization of Application and Network Resource based on Global Load Balancing Strategy in Dynamic Optical Networks," in Proc. OFC/NFOEC, 2012, JTh2A.38.

[17] Yang H., Zhao Y., Zhang J., Wang S., Gu W., Han J., Lin Y., and Lee Y., "Multi-Stratum Resources Integration for Data Center Application based on Multiple OpenFlow Controllers Cooperation," in Proc. OFC/NFOEC, 2013, NTu3F.7.

[18]Zhang J., Yang H., Zhao Y., Ji Y., Li H., Lin Y., Li G., Han J., Lee Y., and Ma T., "Experimental demonstration of elastic optical networks based on enhanced software defined networking (eSDN) for data center application," Opt. Express, vol. 21, no. 22, pp. 26990-27002, 2013. http://dx.doi.org/10.1364/OE.21.026990

[19] Yang H., Zhang J., Zhao Y., Li H., Huang S., Ji Y., Han J., Lin Y., Lee Y., “Cross Stratum Resilience for OpenFlow-enabled Data Center Interconnection with Flexi-Grid Optical Networks," Optical Switching and Networking, Vol. 11, Part A, January 2014, pp. 72-82 http://dx.doi.org/10.1016/j.osn.2013.10.001

\section{Copyright Disclaimer}

Copyright reserved by the author(s).

This article is an open-access article distributed under the terms and conditions of the Creative Commons Attribution license (http://creativecommons.org/licenses/by/3.0/). 